\title{
Genetics of Huntington Disease
}

\author{
S. Mahalingam and L.M. Levy
}

ABBREVIATIONS: $\mathrm{HD}=$ Huntington disease $\mathrm{CAG}=$ cytosine-adenine-guanine

$\mathrm{H}^{\mathrm{s}}$ untington disease (HD) is an autosomal dominant genetic condition that can affect movement and cognition and is progressive and fatal. It results from genetic mutations involving trinucleotide repeats of the huntingtin gene, which encodes the huntingtin protein.

HD is presently the most widely studied genetic neurodegenerative disease that has diagnostic and predictive genetic testing, with the possibility of gene-targeted therapy in the near future.

Neuroimaging can play an important diagnostic and prognostic role in HD by evaluating affected regions of the brain by using techniques such as MR imaging, FDG-PET, MR spectroscopy, and diffusion tensor imaging.

\section{HISTORY OF HUNTINGTON DISEASE}

Huntington disease is a neurodegenerative genetic condition that can affect movement and cognition and is progressive and fatal. While it has been known by various names previously, it obtained its eponym after George Huntington presented an exhaustive description of the clinical manifestation of the disease in $1872 .{ }^{1} \mathrm{Al}-$ though it was called Huntington's chorea for nearly a century, it is now more accurately referred to as Huntington disease, because chorea is neither a constant nor a particularly dominant feature of the disease. HD is an autosomal dominant disease that exhibits complete penetrance, so that all individuals carrying the defective gene will manifest the disease. ${ }^{2}$ The largest number of closely related affected patients comes from interrelated families from Lake Maracaibo in Venezuela. ${ }^{1}$ An extensive project studying these families led to mapping of the gene to the short arm of chromosome 4 at locus 16.3. ${ }^{3}$ The huntingtin protein was later isolated 1993. ${ }^{4}$ HD holds a unique position in the field of medical genetics because it has largely contributed to the understanding of the

From the George Washington University Medical Center, Department of Radiology, Washington, DC.

Please address correspondence to Lucien M. Levy, MD, PhD, George Washington University Medical Center, Department of Radiology, 900 23rd St NW, Washington, DC 20037; e-mail: llevy@mfa.gwu.edu

http://dx.doi.org/10.3174/ajnr.A3772 gene: This was the first genetic disorder to be mapped to a specific locus in the chromosome without prior knowledge of the gene location and one of the first diseases to have prenatal genetic testing made available. ${ }^{1} \mathrm{HD}$ is more common in whites, with an estimated prevalence of $4-10$ per $10,000,{ }^{2}$ and much lower in Asian and African populations. ${ }^{5}$

\section{WHAT ARE THE CLINICAL MANIFESTATIONS OF HUNTINGTON DISEASE?}

Huntington disease has 3 subtypes, with the adult-onset being the most common and the juvenile and infantile varieties being far less prevalent. In adult-onset $\mathrm{HD}$, the disease is characterized by a triad of behavioral, cognitive, and motor features. ${ }^{2}$ Behavioral symptoms often present early as increased irritability, agitation, loss of inhibition, and increased aggression. In a patient without a definite family history, definitive diagnosis with these symptoms is often delayed. However, diagnosis is often easier with evidence of motor symptoms. These include chorea, which can become less pronounced with the onset of rigidity and dyskinesia; motor impersistence (the inability to maintain a sustained voluntary muscle contraction); and loss of fine and gross motor skills, which respectively occur in the early and late phase of the disease. ${ }^{5}$ In general, HD is a devastating and relentlessly progressive disease, which is fatal within 15-20 years of onset. ${ }^{2}$

Juvenile-onset HD and infantile HD account for $<10 \%$ of the disease prevalence. ${ }^{6}$ Rigidity and dyskinesia along with cognitive decline are dominant features with chorea rarely seen. ${ }^{6}$ Regression of motor milestones and poor performance in school are often present at the time of diagnosis.

\section{WHAT ARE THE FUNCTIONS OF THE HUNTINGTIN GENE?}

Although the gene map for huntingtin was discovered many years ago, the exact function of the protein has yet to be confirmed. Given its location in both the cytoplasm and nucleus and its numerous interactions with other proteins, it has been postulated that the huntingtin protein has a regulatory role in transcription 
and intracellular transport. ${ }^{4}$ It is widely expressed in many cell types, with a preferential expression in the brain and testis and, to a lesser, extent in the liver and lungs. ${ }^{7}$ Its distribution in the brain is variable, with high amounts present in the corpus striatum and the cerebral and cerebellar cortices. This protein also has an antiapoptotic role, and cells with mutant or decreased expression of huntingtin undergo early cell dysfunction and death. Expansion of the cytosine-adenine-guanine (CAG) repeats causes increased polyglutamine in the huntingtin gene, with CAG corresponding to the 3 DNA bases, cytosine-adenine-guanine. This leads to the formation of abnormal nuclear and cytoplasmic intracellular inclusions or aggregates, which dysregulate cell homeostasis and promote cell death ${ }^{4,8}$

\section{WHAT IS THE SIGNIFICANCE OF THE HUNTINGTIN PROTEIN?}

The normal huntingtin protein has fewer than 27 CAG repeats that encode for polyglutamine. Individuals with CAG repeats of 27-35 (intermediate alleles) ${ }^{9}$ will not manifest the disease, though intermediate alleles possess the potential to expand into an allele range of $\geq 36$ in subsequent generations. Those with $\geq 36 \mathrm{CAG}$ repeats are affected with $\mathrm{HD}$ and will manifest the disease.

The greater the number of the CAG repeats, the earlier is the onset of the disease. With each generation, there is an increase in the CAG triplet expansion, leading to "anticipation" of the disease. ${ }^{5}$ Thus, patients with infantile- and juvenile-onset $\mathrm{HD}$ will possess a large number of repeats in their alleles and earlier onset compared with their parents, from whom they inherited the disease. ${ }^{10}$

\section{WHAT IS THE SIGNIFICANCE OF THE INTERMEDIATE ALLELE AND THE ROLE OF SEX?}

The intermediate allele containing the CAG repeat of 27-35 shows a greater degree of instability and a propensity for expansion during spermatogenesis compared with oogenesis. ${ }^{9}$ Thus a male with the intermediate allele has a higher probability of producing an offspring with the HD allele containing $\leq 36$ CAG repeats than a female with an intermediate allele. In addition, juvenile-onset $\mathrm{HD}$ and infantile $\mathrm{HD}$ have a higher percentage of paternal inheritance compared with maternal inheritance. ${ }^{6}$

\section{DIAGNOSTIC TESTING}

In individuals with a strong family history of HD and evidence of appropriate symptoms, genetic testing is often unnecessary. However, in situations in which the diagnosis is not apparent or a positive family history is unavailable, negative testing results can conclusively rule out the disease and allow the pursuit of alternative differential diagnoses.

\section{IS THERE PREDICTIVE TESTING AVAILABLE FOR HUNTINGTON DISEASE?}

Considering the prolonged and difficult course of this fatal disease, obtaining genetic testing is a significant decision for the patient. Presently, predictive testing is offered after due review of the individual's mental status and available psychological support to prevent any adverse outcomes. ${ }^{5}$ In general, predictive testing is used by a person with a family history of HD before significant life-changing events such as marriage or pregnancy. ${ }^{3}$ Prenatal diagnostic testing is also available to determine the HD allele status of the fetus. However, predictive testing cannot be used to forecast the age at onset because it has a high degree of variability. ${ }^{2}$ Thus, even though individuals with larger numbers of repeats have earlier onset, the number of repeats cannot predict the age of onset. ${ }^{3}$

\section{WHAT ARE THE ROLES OF STRUCTURAL AND FUNCTIONAL NEUROIMAGING IN HD?}

Neuroradiology can play a diagnostic and prognostic role in the evaluation of HD. In individuals with a strong family history of $\mathrm{HD}, \mathrm{MR}$ volumetric analysis can predict loss of striatal volume as early as 20 years before the appearance of motor symptoms. ${ }^{11}$ In the clinical symptomatic phase, MR imaging and CT can identify caudate atrophy and the consequent dilation of the frontal horns. Cortical and subcortical atrophy are heterogeneous and appear later than striatal atrophy. Loss of striatal volume can serve as a biomarker for predicting the course of the disease because it is constant throughout. ${ }^{2}$ FDG-PET can reveal a decrease in the metabolism of glucose in the striatum in the early phase of the disease, and subsequently in the cortex. A recent study suggested that glucose hypometabolism in the cortex can serve as a marker for faster disease progression. ${ }^{12}$ MR spectroscopy can be used to detect decreases in $\mathrm{N}$-acetylaspartate and creatine in the caudate and putamen along with decreased putaminal glutamate during the preclinical phase. Also, MR spectroscopy has demonstrated a correlation between disease severity and levels of $\mathrm{N}$-acetylaspartate, creatine, and glutamate, and these may be useful as future biomarkers. ${ }^{13}$ Diffusion tensor imaging has also been useful in demonstrating the degeneration of white matter tracts, which can occur early in the course of the disease. ${ }^{14,15}$

\section{CONCLUSIONS}

Huntington disease is the most widely studied genetic neurodegenerative disease that has available diagnostic and predictive genetic testing, with the possibility of gene-targeted therapy in the near future. Valuable insights provided by genetic research in HD have also strengthened the understanding of other neurodegenerative disorders such as Alzheimer disease, with increased hope for more definitive therapeutic strategies.

\section{REFERENCES}

1. Bates GP. History of genetic disease: the molecular genetics of Huntington disease: a history. Nat Rev Genet 2005;6:766-73

2. Ross CA, Tabrizi SJ. Huntington's disease: from molecular pathogenesis to clinical treatment. Lancet Neurol 2011;10:83-98

3. Myers RH. Huntington's disease genetics. NeuroRx 2004;1:255-62

4. Borrell-Pagès $M$, Zala $D$, Humbert $S$, et al. Huntington's disease: from huntingtin function and dysfunction to therapeutic strategies. Cell Mol Life Sci 2006;63:2642-60

5. Walker FO. Huntington's disease. Lancet 2007;369:218-28

6. Koutsis G, Karadima G, Kladi A, et al. The challenge of juvenile Huntington disease: to test or not to test. Neurology 2013;80:990-96

7. Trottier Y, Devys D, Imbert G, et al. Cellular localization of the Huntington's disease protein and discrimination of the normal and mutated form. Nat Genet 1995;10:104-10

8. Bates G. Huntingtin aggregation and toxicity in Huntington's disease. Lancet 2003;361:1642-44

9. Semaka A, Creighton S, Warby S, et al. Predictive testing for HunAJNR Am J Neuroradiol 35:1070-72 Jun 2014 www.ajnr.org

1071 
tington disease: interpretation and significance of intermediate alleles. Clin Genet 2006;70:283-94

10. Tabrizi SJ, Langbehn DR, Leavitt BR, et al. Biological and clinical manifestations of Huntington's disease in the longitudinal TRACK-HD study: cross-sectional analysis of baseline data. Lancet Neurol 2009;8:791-801

11. Klöppel S, Henley SM, Hobbs NZ, et al. Magnetic resonance imaging of Huntington's disease: preparing for clinical trials. Neuroscience 2009;164:205-19

12. Shin H, Kim MH, Lee SJ, et al. Decreased metabolism in the cerebral cortex in early-stage Huntington's disease: a possible biomarker of disease progression? J Clin Neurol 2013;9:21-25
13. Versluis MJ, van der Grond J, van Buchem MA, et al. High-field imaging of neurodegenerative diseases. Neuroimaging Clin N Am 2012;22:159-71, ix

14. Bohanna I, Georgiou-Karistianis N, Sritharan A, et al. Diffusion tensor imaging in Huntington's disease reveals distinct patterns of white matter degeneration associated with motor and cognitive deficits. Brain Imaging Behav 2011;5:171-80

15. Rosas HD, Tuch DS, Hevelone ND, et al. Diffusion tensor imaging in presymptomatic and early Huntington's disease: selective white matter pathology and its relationship to clinical measures. Mov Disord 2006;21:1317-25 